\title{
Disabled, but at What Cost?
}

\section{An Examination of Wheelchair Routing Algorithms}

\author{
Benjamin Tannert \\ University of Bremen \\ Bremen, Germany \\ btannert@uni-bremen.de
}

\author{
Johannes Schöning \\ University of Bremen \\ Bremen, Germany \\ schoening@uni-bremen.de
}

\begin{abstract}
Platforms like Google Maps or Bing Maps are used by a large number of users to find the shortest path to their destinations. While these services mainly focus on supporting drivers and pedestrians, first services exist that support wheelchair users. Routing algorithms for wheelchair users try to avoid obstacles like stairs or bollards and optimize on criteria like surface properties and slope of the route. In this study, we undertake the first controlled examination of wheelchair routing approaches. By analyzing three routing platforms, including two wheelchair routing algorithms and three pedestrian routing algorithms, across fifteen major cities in Germany, our results highlight that the routes for wheelchair users are significantly longer and partially also more complex than those for pedestrians. In addition, we show that today's pedestrian routing algorithms also output very diverse routes.
\end{abstract}

\section{Author Keywords}

Accessibility; HCI; Disability; Wheelchair Users; Pedestrian Navigation; Routing; City Planning.

\section{ACM Classification Keywords}

H.5.m. Information interfaces and presentation (e.g., HCI): Miscellaneous;

\section{INTRODUCTION \& RELATED WORK}

Besides a large number of unreported cases, more than 65 million people in the world need a wheelchair on a daily basis [13]. In Germany, as per the Federal Statistical Office $[17],$,1.5 million people need a wheelchair every day. This is 2.7 percent of the whole population of Germany.

Permission to make digital or hard copies of all or part of this work for personal or classroom use is granted without fee provided that copies are not made or distributed for profit or commercial advantage and that copies bear this notice and the full citation on the first page. Copyrights for components of this work owned by others than the author(s) must be honored. Abstracting with credit is permitted. To copy otherwise, or republish, to post on servers or to redistribute to lists, requires prior specific permission and/or a fee. Request permissions from Permissions@acm.org.

MobileHCI '18, September 3-6, 2018, Barcelona, Spain

(C) 2018 Copyright is held by the owner/author(s). Publication rights licensed to ACM.

ACM ISBN 978-1-4503-5898-9/18/09 ..\$15.00

https://doi.org/10.1145/3229434.3229458

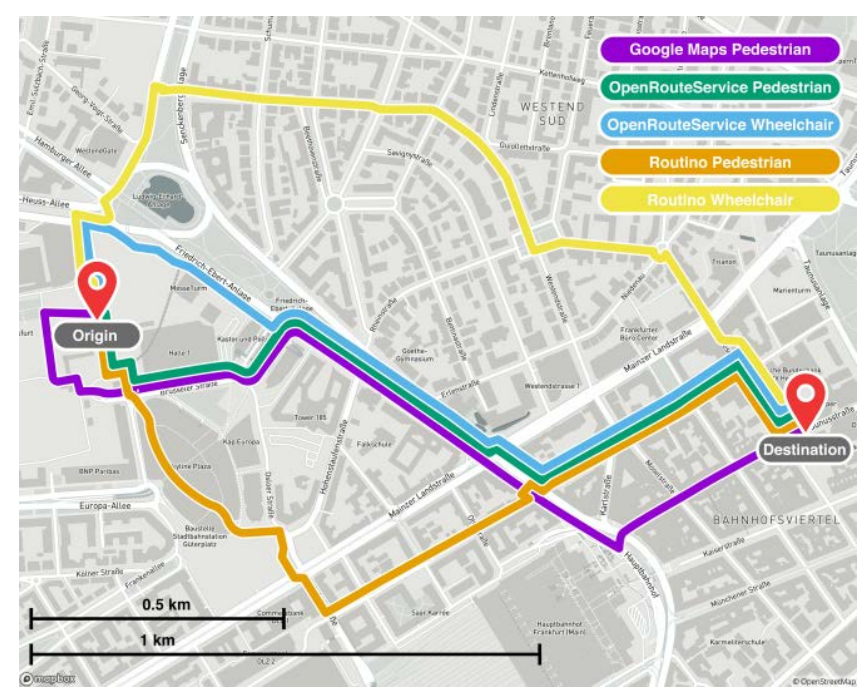

Figure 1: Routes for pedestrians (purple, green, orange) and wheelchair users (blue, yellow) calculated by different routing platforms for an origin-destination pair in Frankfurt, Germany. Base map (C) OSM.

Various mobile technologies, apps and web platforms can support wheelchair users to master their daily lives. For example, Wheelmap (http://wheelmap.org) provides crowdsourced accessibility information for buildings, points of interests (POIs) and restaurants based on OpenStreetMap (OSM).

The Wheelmap service uses a traffic light metaphor to indicate if a building is easily accessible or not. Google also recently announced their decision to crowd-source accessibility information for buildings [1] to be integrated into Google Maps.

Besides accessibility information, obstacles along a route are crucial for wheelchair users. While some obstacles are obvious (e.g. steps or pillars), some others are often only considered by disabled people (e.g. slope of sidewalks or different surface properties like sand or cobblestone, which are difficult to overcome in a wheelchair). Various approaches analyzed the accessibility of sidewalks with sensors and technical equipment such as depth cameras or acceleration sensors $[3,4,5,6,9,12,16,19,20]$. 
Besides these (semi-) automated approaches, Hara et al. [2] explored the use of Amazon Mechanical Turk (AMT) workers for classifying the accessibility of sidewalks for wheelchair users. They compared the differences in the annotation of the AMT workers and the experts (wheelchair users) and found that even untrained personnel could easily identify problems in the image of sidewalks.

Today, first routing services exist that help to guide wheelchair users from an origin to a destination $[10,11$, 17]. These services take numerous variables into account to provide routes that are easy for wheelchair users to follow. Most commonly, they avoid obstacles and barriers such as stairs or bollards, utilize the surface properties, the slope, and the height of the pavement edges to calculate routes particularly suited for wheelchair users. Karimi et al. [8] provide a good overview of requirements and components needed for a routing service that can assist disabled people.

In this study, we undertake the first controlled examination of these wheelchair routing services and report on the following three contributions:

- We found that significant differences exist between routes for pedestrian and wheelchair users within same areas. For example, in Frankfurt, Germany, routes (between 1.5 and $2.0 \mathrm{~km}$ straightline distance between origin and destination) for wheelchair users were on average nearly double the length of those for pedestrians and were also more complex (e.g. in terms of \# of turns).

- Furthermore, a notable difference exists between cities. While we observed a large difference in Frankfurt, the difference between routes for pedestrian and wheelchair users differed by just 1 $\%$ in Hamburg (again for routes between 1.5 and $2.0 \mathrm{~km}$ straight-line distance between origin and destination and routes calculated with OpenRouteService). Therefore, our results provide a novel way to benchmark cities with regard to the wheelchair accessibility.

- We also noticed that pedestrian routing algorithms calculate very different routes in terms of their complexity even though they provide routes of similar length. For example, Google Maps tries to minimize the number of turns to reduce the complexity of the calculated routes even so this involved detours.

\section{STUDY}

In our study, we investigated three different routing platforms, namely Google Maps (https://www.google.de/maps), OpenRouteService (https://www.openrouteservice.org) and Routino (https://www.routino.org). While Google Maps uses proprietary data, OpenRouteService (ORS) and Routino both rely on data from OpenStreetMap (OSM) (https://www.openstreetmap.de/) for their calculations and both offer dedicated routing algorithms for wheelchair

\begin{tabular}{|l|c|c|c|c|}
\hline & Speed & $\begin{array}{c}\text { To be } \\
\text { Paved }\end{array}$ & $\begin{array}{c}\text { Avoid } \\
\text { Steps }\end{array}$ & Base Data \\
\hline Rou_Ped & $4 \mathrm{~km} / \mathrm{h}$ & $50 \%$ & No & OSM \\
\hline Rou_Wheel & $4 \mathrm{~km} / \mathrm{h}$ & $90 \%$ & Yes & OSM \\
\hline ORS_Ped & $6 \mathrm{~km} / \mathrm{h}$ & $0 \%$ & No & OSM \\
\hline ORS_Wheel & $8 \mathrm{~km} / \mathrm{h}$ & $100 \%$ & Yes & OSM \\
\hline Google_Ped & $5 \mathrm{~km} / \mathrm{h}$ & - & N/A & proprietary \\
\hline
\end{tabular}

Table 1: Overview of the main criteria of the algorithms.

users. Google Maps provide routes for different modes of locomotion but not for wheelchair users.

The ORS algorithms use default settings to calculate the routes for pedestrians (ORS_Ped) and wheelchair users (ORS Wheel). ORS Ped assumes a speed of $6 \mathrm{~km} / \mathrm{h}$ and ORS_Wheel uses $8 \mathrm{~km} / \mathrm{h}$. And while in the ORS_Wheel the whole route has to be paved, the surface is not taken into account in ORS_Ped.

Similarly, the ORS_Wheel algorithm avoids steps, while ORS Ped does not take this feature into consideration. Additionally, ORS_Wheel takes the incline of route segments (max. $6 \%$ incline) and the height of sloped curbs (max. height of $6 \mathrm{~cm}$ ) into account. A detailed description of the ORS routing algorithms can be found here [14].

In contrast to the ORS algorithms, the Routino algorithm for pedestrians (Rou Ped) and Routino for wheelchair users (Rou Wheel) both assume the speed of $4 \mathrm{~km} / \mathrm{h}$ for their calculations. While in the Rou Wheel $90 \%$ of the route has to be paved, only $50 \%$ needs to be paved in Rou_Ped. Similar to ORS_Wheel, Rou_Wheel avoids steps. The detailed description of the Routino algorithms can be found here [15].

Given the fact that Google uses proprietary algorithm and data, we cannot provide detailed information on the implementation of the Google_Ped algorithm. The only available information is that the average speed is assumed to be $5 \mathrm{~km} / \mathrm{h}$ to calculate the time of travel.

Table 1 provides a comparison of the main criteria used by the algorithms analyzed in this paper. Figure 1 shows an example of different routes calculated by the five algorithms used in our study for one origin-destination pair in Frankfurt, Germany.

\section{Framework}

To evaluate the 5 routing algorithms (Google_Ped, ORS Ped, ORS Wheel, Rou Ped, Rou Wheel), we extended a framework developed by Johnson et al. [7] and integrated all five routing algorithms. Johnson et al. used their framework to investigate externalities that arise with three common approaches to the fastest path option ("beauty", "safety" and "simplicity") for car-based routing algorithms across four cities around the globe, namely London, Manila, San Francisco and New York. They used 
a) Average Route Length AllCities

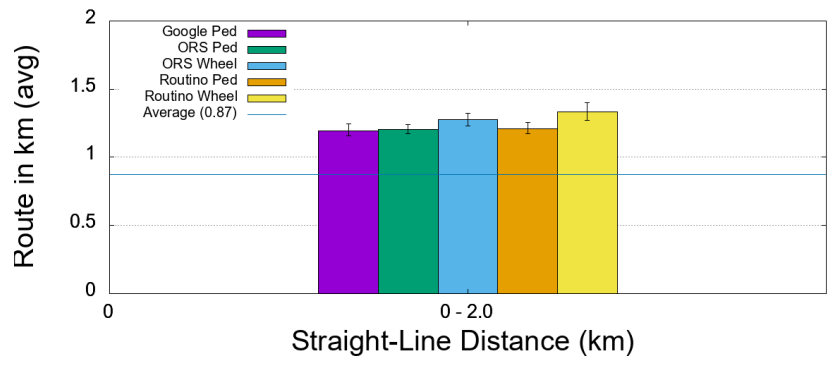

c) Average Route Length Frankfurt

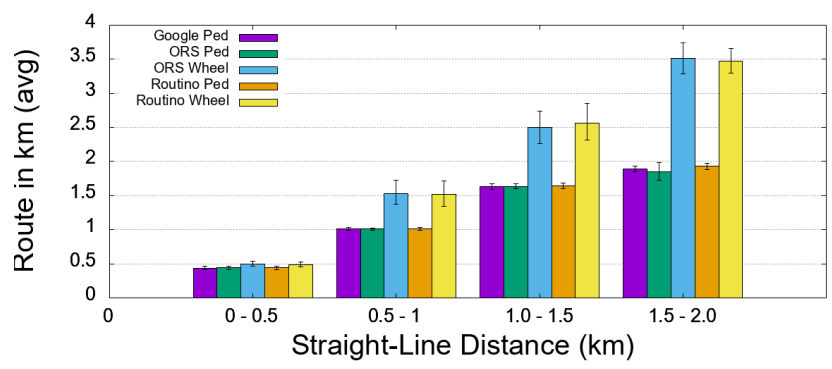

b) Average Route Length AllCities

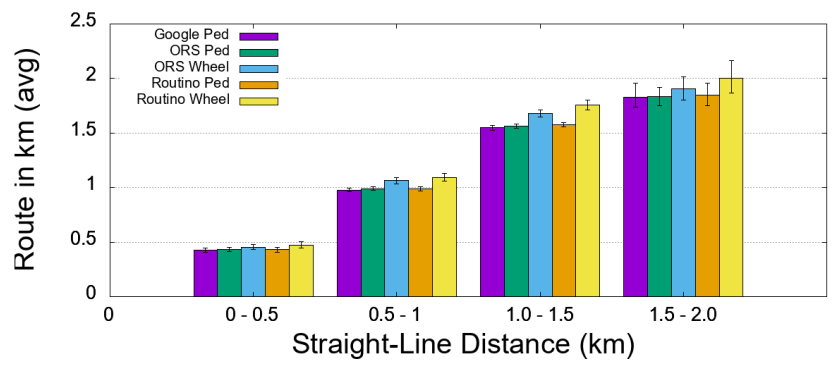

d) Average Route Length Hanover

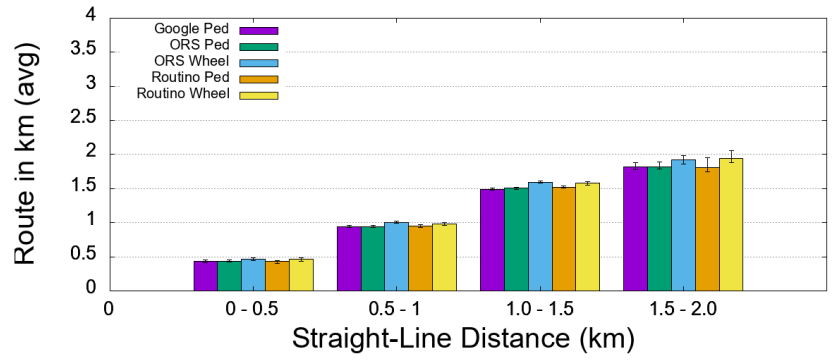

Figure 2: Average route length a) across all cities b) grouped in distance bins and c) in Frankfurt and d) Hanover.

around 1000 origin-destination pairs for each city to compare the effects of the different routing options. For the origin and destination pairs, they used information about the most common pathways of taxi companies and generated random points in the city.

\section{Origin-Destination Pairs}

In order to compare the outcome provided by the different routing platforms, we had to identify a set of representative origin-destination pairs for all fifteen cities. As no public data was available for wheelchair routes in those cities, we calculated origin-destination pairs between POIs and public restrooms for disabled people. As confirmed by wheelchair users, these pairs describe a set of typical routes.

We selected the 15 biggest German cities namely, Berlin (BER), Bremen (HB), Cologne (COL), Dortmund (DOR), Dresden (DRE), Düsseldorf (DUE), Erfurt (ERF), Essen (ESS), Frankfurt (FRA), Hamburg (HH), Hanover (HAN), Leipzig (LEI), Munich (MUN), Nuremberg (NUR) and Stuttgart (STU). One of the cities is the hometown of one of the co-authors, who also uses a wheelchair, which offers the possibility to use his knowledge to compare the quality of the calculated routes. To generate the origin-destination pairs we first gathered data on the location of public restrooms for disabled people from www.myhandicap.de. Then, we used the Google Maps API to identify POIs in a radius of $2 \mathrm{~km}$ around these public restrooms. The POIs, e.g. parks or restaurants, are places from where wheelchair users would usually need to drive to a restroom. The Google Maps API provides up to 200 POIs around each location. Using this process, we generated 267.421 origin- destination pairs for all 15 cities. We extracted 2715 pairs for every city with the same average straight-line distance and same variance to be comparable across all 15 cities. For further analysis, we grouped these pairs into four classes of routes that have a straight-line distance of 0.0-0.5 km, 0.5$1.0 \mathrm{~km}, 1.0-1.5 \mathrm{~km}$ and $1.5-2.0 \mathrm{~km}$.

\section{RESULTS}

In order to derive evidence on the statistical differences between the routing alternatives per city and the summary of them (in terms of length and number of turns), we used a one-way ANOVA with Bonferroni correction. The significance threshold was set to $\mathrm{p}<0.05$.

Figure 2a summarizes the average length of the routes of all five routing algorithms across the fifteen cities (40.725 origin-destination pairs for all cities, 2715 routes per city). There is a significant difference between all pairs of routing algorithms with the exception of the pair ORS_Ped and Rou_Ped, which is somehow expected as both pedestrian routing algorithms are operating on OSM data. We further calculated the effect size of each pair with a significant difference to analyze the impact of it. The results of this analysis showed that the effect sizes were negligible.

Figure $2 \mathrm{~b}$ shows the average route length grouped into classes by the straight-line distance between origin and destination. The chart illustrates that the difference of the length between the pedestrian and the wheelchair algorithms grow with the length of the straight-line distance. 
For the classes $0-0.5 \mathrm{~km}, 0.5-1.0 \mathrm{~km}$ and $1.0-1.5 \mathrm{~km}$ there are no significant differences, but for the class $1.5-2.0 \mathrm{~km}$ all differences between routing algorithms are significant (again with the exception of the pair ORS Ped and Rou_Ped). To summarize, Figures $2 \mathrm{a}$ and $2 \mathrm{~b}$ show that the routes for wheelchair users are indeed longer than those for pedestrians. Moreover, this difference increases as the straight-line distance between origin and destination point grows. Google_Ped generates the shorter routes compared to all other 4 routing algorithms. Routino Wheel generates longer route lengths than the pedestrian algorithms in all fifteen cities.

We observe a continuous increase in the gap between the average wheelchair and pedestrian route lengths, with the rise of the straight-line measure between origin and destination. We also found that Google Ped is a good representative of the other pedestrian algorithms, and for straight-line distances (average of all cities) between 0.0$0.5 \mathrm{~km}$, the difference in route length of Google_Ped and Rou_Wheel is approximately $50 \mathrm{~m}$. For the next class, this difference is $120 \mathrm{~m}$ and for $1.0-1.5 \mathrm{~km}$ it is $250 \mathrm{~m}$. For the last class between $1.5 \mathrm{~km}$ and $2.0 \mathrm{~km}$ the difference increases to $300 \mathrm{~m}$.

\section{Wheelchair vs. Pedestrian Routing across Cities}

We expected that all three pedestrian routing algorithms (Google_Ped, ORS_Ped, and Rou_Ped) generate shorter routes as both algorithms for wheelchair users (ORS_Wheel and Rou_Wheel). Therefore, we decided not only to compare the groups of pedestrian and wheelchair algorithms in general, but also between cities. We further analyzed the differences between the five algorithms across the 15 cities.

As can be seen in figure 2c in FRA, for the straight-line distance class of 1.5-2.0 km, there is a significant average difference of $1,5 \mathrm{~km}$ between Google Ped and Rou Wheel as well as 1,6 km between Google_Ped and ORS_Wheel.

As can be seen in figure $2 \mathrm{~d}$ in HAN, the differences are around $100 \mathrm{~m}$ on average between Google_Ped and ORS Wheel and $120 \mathrm{~m}$ between Google Ped and Rou_Wheel, but still significant. Frankfurt was a representative city for a rather high difference between the algorithms, whereas Hanover was an example for a city with rather small differences between the five routing algorithms.

When comparing other pairs of algorithms, we found similar trends. For example, while the average difference of ORS Ped and ORS Wheel for 1.5-2.0 km origindestination pairs is around $100 \mathrm{~m}$ in HAN, it is around 2.0 $\mathrm{km}$ in FRA. We observe similar patterns between Rou_Ped and Rou_Wheel. In the group of the fifteen cities, FRA is special because, the route length of the wheelchair algorithms is much longer than in the other cities.

\section{Route Complexity}

Finally, we examined the complexity of the routes that were calculated by the five routing algorithms. We analyzed if there is a significant difference between the pedestrian and wheelchair algorithms in terms of the overall number of turns for each route.

Figure 3 shows the averages number of turns for the cities HAM, LEI, DOR and FRA. Here it can be seen that the complexity is not always higher for wheelchair users than for pedestrians. For example, in DOR the ORS Ped algorithm is more complex than ORS_Wheel for routes between 1.0-1.5 km, but for routes between 1.5-2.0 km the ORS_Wheel algorithm indicates a higher number of turns. The Rou_Ped and the Rou_Wheel perform in a similar way in FRA for the equivalent route lengths. Instead in HAM the higher complexity does not switch between the pedestrian and wheelchair routes, but it is different between the providers. Here the ORS algorithms show always that the routes for wheelchair users are more complex, whereas the Rou algorithms indicate that the pedestrians have more turns on their routes.

We inferred that the difference in the number of turns depends strongly on the provider (Google, ORS, Rou). In comparison to Google_Ped, the ORS_Ped algorithm require more turns for the $1.5-2.0 \mathrm{~km}$ class in all cities; $\mathrm{HH}$ (1.9), LEI (14.2), DOR (14.1) and FRA (13.5). The Rou_Ped seems to generate the routes not optimizing for a low number of turns and complexity. It includes twice as many turns as the ORS Ped and many times more turns than Google_Ped. For the straight-line distance section of $1.5 \mathrm{~km}$ and $2.0 \mathrm{~km}$ the difference in the number of turns between Google_Ped and Rou_Ped is in HH 46.5, in LEI 40.9, in DOR $3 \overline{9} .3$ and in FRA 37.8 .

\section{Wheelchair Routing Differences}

As can be seen in figure 4, the fifteen analyzed cities are slightly different in terms of wheelchair accessibility. For every of the 15 cities the figure shows the averaged route length of all routes generated for wheelchair users (ORS_Wheel and Rou_Wheel). Thus, it can be seen in which cities the route lengths are similar and in which one of the algorithms perform different. On the other hand, the average route lengths of ORS_Wheel are sometimes similar to those of Rou_Wheel but are often shorter even though both use the same data e.g about the surface.

If we look at the pedestrian algorithms (Google Ped, ORS Ped, and Rou Ped), it is obvious that the average length of the routes is very similar. Only for higher straightline distances in FRA, Rou_Ped generates longer routes than the other pedestrian algorithm. For the $1.5-2.0 \mathrm{~km}$ class, the average route length is approximately $0.15 \mathrm{~km}$ longer. Although the average route lengths of the pedestrian algorithms are very similar, the complexity and therefor the number of turns of the routes are different (Figure 3). It can thus be concluded that the route length is similar, but the way is different. 
Average Turns Hamburg

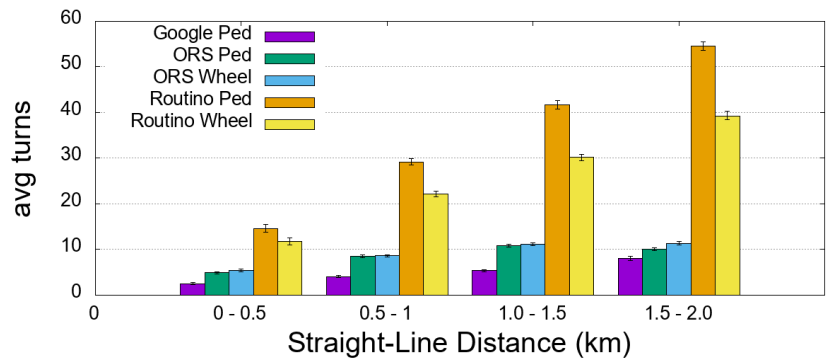

Average Turns Dortmund

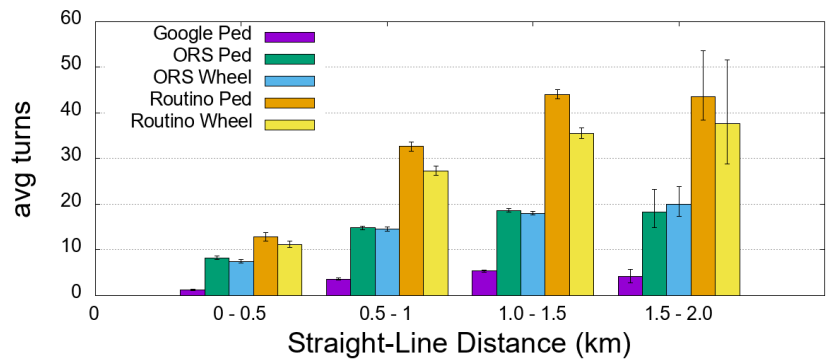

Average Turns Leipzig

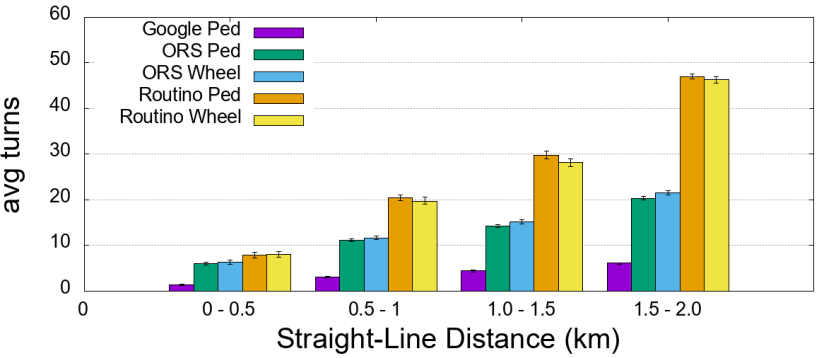

Average Turns Frankfurt

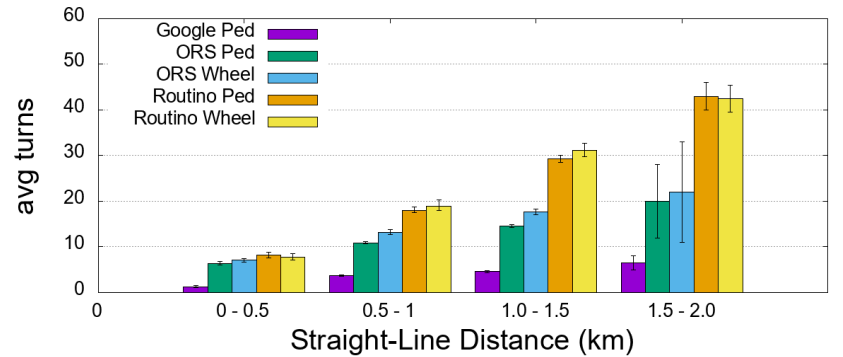

Figure 3: Average number of turns across the five routing algorithms for four cities grouped by straight-line distance.

\section{DISCUSSION}

The results of our analysis show that the accessibility of cities can differ significantly.

\section{Societal Impact of Wheelchair Routing}

The results for FRA illustrate a weak implementation of accessibility standards, which has a direct impact on lives of many wheelchair users. This is accentuated by the long routes generated by ORS_Wheel and Rou_Wheel. The difference between Rou_Ped and Rou_Wheel includes obstacles that wheelchair users have to avoid. A detailed survey of such obstacles could be useful not only to wheelchair users but also other stakeholders.

\section{Complexity of Routing Algorithms}

Trying to reach a destination from a given origin point can be very difficult if a turn is missed. Figure 3 shows that Google_Ped is trying to reduce the complexity of the route by minimizing the number of turns to prevent wrong turns. Compared to Google_Ped the algorithms of Routino (Rou_Ped and Rou_Wheel) produce more complex routes and therefore have more decision points, where users can make mistakes. In addition to that, the ORS algorithms (ORS_Ped and ORS_Wheel) generate the double number of turns as the Google algorithm. Overall, the complexity of a route is more depending on the provider than the modality (pedestrians and wheelchair users).

\section{Limitations}

To generate origin destination pairs, we used only randomly generated pairs (between public restrooms for disabled people and POIs). It would be interesting to include more realistic data into our framework, but no such data exists that captures typical routes of wheelchair users across multiple cities (similar to the taxi data used by Johnson et al. [7]) according to the Federal Statistical Office of Germany as well as the building authorities and departments of town planning of the 10 largest cities in Germany. This data would not only be useful to be plugged in our framework, but also for more general planning activities.

The fifteen cities examined in our study are scattered over Germany, hence their geographical topology can differ due to various causes. However, we could still inspect differences in routing algorithms and analyze the results in respect to the wheelchair accessibility issue.

In general, the more spatial data and attributes are available to calculate the route, the better the algorithms of the routing platforms can perform. The data to be used by the pedestrian routing algorithms is mostly complete and requires less attributes information. However, this is not the case with the algorithms for wheelchair users that have to use incomplete data for different parts of the city collected mostly by volunteers. Therefore, missing or incomplete information can also lead to failures in wheelchair navigation, producing longer and/or inaccessible routes. 


\section{Length of Routes for Wheelchair Users of all Cities}

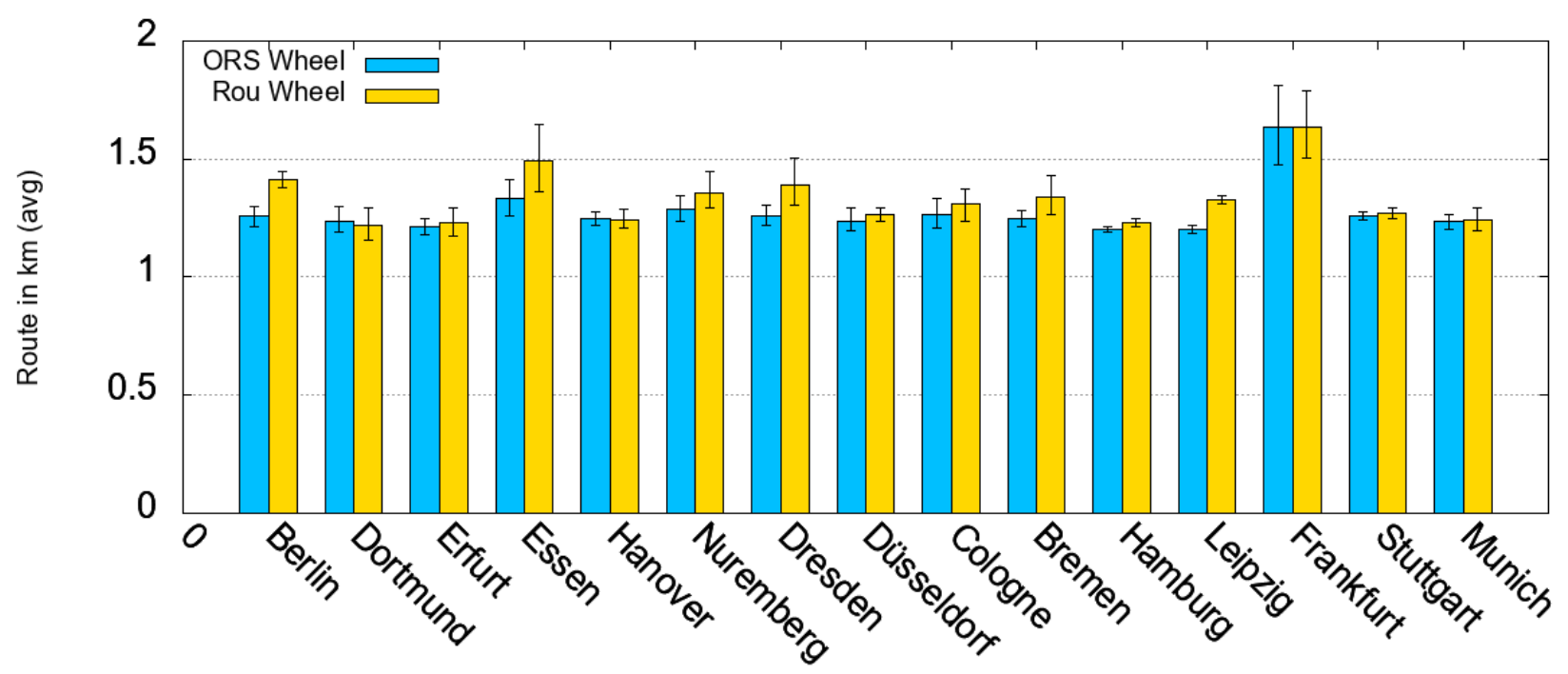

Figure 4: Average length of routes for wheelchair users.

\section{CONCLUSION}

By analyzing three pedestrian routing platforms, including two wheelchair routing algorithms, across fifteen major cities in Germany we show that the routes for wheelchair users are significantly longer and partially also more complex than those for pedestrians. As this could be due to missing attribute information the wheelchair routing algorithms rely now, we manually investigated those cases in the city of Bremen. We found out that, even so attribute information is missing, many barriers still exist that could be removed by decision makers to minimize route lengths for wheelchair users. In addition, we as technologist can help to collect missing attribute information to improve the route generation of wheelchair routing algorithms. Automatic and semi-automatic approaches, similar to the ones proposed by $[2,9,16,20]$, could be used to fill this gap.

\section{ACKNOWLEDGMENTS}

We would like to thank Ankit Kariryaa and Reuben Kirkham for their valuable comments and input on this paper. Furthermore, we thank Isaac Johnson for the help adapting his framework and Brent Hecht for his support in the beginning of this study. This work is also partially supported by the project "InWi - Inklusion in der Wissenschaft" and by the Volkswagen Stiftung through a Lichtenberg Professorship.

\section{REFERENCES}

1. Sasha Blair-Goldensohn. 2017. A better world for wheels on Google Maps.

https://www.blog.google/products/maps/better-worldwheels-google-maps/
2. Kotaro Hara, Vicki Le, and Jon Froehlich. 2013. Combining crowdsourcing and google street view to identify street-level accessibility problems. In Proceedings of the SIGCHI Conference on Human Factors in Computing Systems (CHI '13). ACM, New York, NY, USA, 631-640. DOI: https://doi.org/10.1145/2470654.2470744

3. Catherine Holloway \& Nick Tyler (2013) A microlevel approach to measuring the accessibility of footways for wheelchair users using the Capability Model, Transportation Planning and Technology, 36:7, 636-649, DOI: 10.1080/03081060.2013.845434

4. Yusuke Iwasawa, Koya Nagamine, Yutaka Matsuo, and Ikuko Eguchi Yairi. 2015. Road Sensing: Personal Sensing and Machine Learning for Development of Large Scale Accessibility Map. In Proceedings of the 17th International ACM SIGACCESS Conference on Computers \& Accessibility (ASSETS '15). ACM, New York, NY, USA, 335-336. DOI: https://doi.org/10.1145/2700648.2811366

5. Yusuke Iwasawa, Kouya Nagamine, Ikuko Eguchi Yairi, Yutaka Matsuo, Toward an Automatic Road Accessibility Information Collecting and Sharing Based on Human Behavior Sensing Technologies of Wheelchair Users, In Procedia Computer Science, Volume 63, 2015, Pages 74-81, ISSN 1877-0509, https://doi.org/10.1016/j.procs.2015.08.314.

6. Nor Haslina Ja'afar, Asiah Abdul Rahim, Nur Amirah Abd. Samad \& Che Raiskandar Che Rahim. 2017. Sidewalk accessibility at Meleka's traditional street for people with disabilities. In Planning Malaysia: Journal 
of the Malaysian Institute of Planners. Volume 15 Volume 1 (2017), 389 - 396. Malaysian Institute of Planners. Petaling Jaya, Selangor Darul Ehsan. DOI: http://dx.doi.org/10.21837/pmjournal.v15.i6.255

7. I. Johnson, J. Henderson, C. Perry, J. Schöning, and B. Hecht. 2017. Beautiful...but at What Cost?: An Examination of Externalities in Geographic Vehicle Routing. Proc. ACM Interact. Mob. Wearable Ubiquitous Technol. 1, 2, Article 15 (June 2017), 21 pages. DOI: https://doi.org/10.1145/3090080

8. Hassan A. Karimi, Lei Zhang \& Jessica G. Benner (2014) Personalized accessibility map (PAM): a novel assisted wayfinding approach for people with disabilities, Annals of GIS, 20:2, 99-108, DOI: $10.1080 / 19475683.2014 .904438$

9. Reuben Kirkham, Romeo Ebassa, Kyle Montague, Kellie Morrissey, Vasilis Vlachokyriakos, Sebastian Weise, and Patrick Olivier. 2017. WheelieMap: an exploratory system for qualitative reports of inaccessibility in the built environment. In Proceedings of the 19th International Conference on HumanComputer Interaction with Mobile Devices and Services (MobileHCI '17). ACM, New York, NY, USA, Article 38, 12 pages. DOI: https://doi.org/10.1145/3098279.3098527

10. N. P. Kozievitch, L. D. A. Almeida, R. Dutra da Silva and R. Minetto, "An alternative and smarter route planner for wheelchair users: Exploring open data," 2016 5th International Conference on Smart Cities and Green ICT Systems (SMARTGREENS), Rome, 2016, pp. 1-6.

11. Kirill Kulakov, Anton Shabaev, and Irina Shabalina. 2015. The route planning services approach for people with disability. In Proceedings of the 17th Conference of Open Innovations Association FRUCT (FRUCT'17), Sergey Balandin, Tatiana Tyutina, and Ulia Trifonova (Eds.). FRUCT Oy, Helsinki, Finland, Finland, Pages 14, 7 pages. DOI: https://doi.org/10.1109/FRUCT.2015.7117977

12. Q. Mourcou, A. Fleury, P. Dupuy, B. Diot, C. Franco and N. Vuillerme, "Wegoto: A Smartphone-based approach to assess and improve accessibility for wheelchair users," 2013 35th Annual International Conference of the IEEE Engineering in Medicine and Biology Society (EMBC), Osaka, 2013, pp. 1194-1197.

13. Newdisability. Wheelchair Statistics: How Many Wheelchair Users Are There? http://www.newdisability.com/wheelchairstatistics.htm

14. ORS. OpenRouteService-docs https://github.com/GIScience/openrouteservice-docs

15. Routino. Routino: Algorithm. https://www.routino.org/documentation/algorithm.html

16. Nina Runge, Pavel Samsonov, Donald Degraen, and Johannes Schöning. 2016. No more Autobahn!: Scenic Route Generation Using Googles Street View. In Proceedings of the 21st International Conference on Intelligent User Interfaces (IUI '16). ACM, New York, NY, USA, 147-151. DOI: https://doi.org/10.1145/2856767.2856804

17. A. S. Sobeck, H. J. Miller. 2006. U-Access: a webbaesd system for routing pedestrians of differing abilities. Journal of Geographical Systems 8, 2: 269287.

18. Statistisches Bundesamt https://www.destatis.de/DE/Publikationen/Thematisch/ Gesundheit/BehinderteMenschen/SozialSchwerbehinde rteKB5227101159004.pdf?_blob=publicationFile

19. Thomas Stockx, Brent Hecht, and Johannes Schöning. 2014. SubwayPS: towards smartphone positioning in underground public transportation systems. In Proceedings of the 22nd ACM SIGSPATIAL International Conference on Advances in Geographic Information Systems (SIGSPATIAL '14). ACM, New York, NY, USA, 93-102. DOI: http://dx.doi.org/10.1145/2666310.2666396

20. Alexander Zipf, Amin Mobasheri, Adam Rousell and Stefan Hahmann. 2016. Crowdsourcing for individual needs - the case of routing and navigation for mobilityimpaired persons. In: European Handbook of Crowdsourced Geographic Information. Pp.325-337. London: Ubiquity Press. 